\title{
Foreign Body in Ear, Nose and Throat: Experience in a Tertiary Hospital
}

\author{
Ritam Ray • Manatosh Dutta • Manoj Mukherjee • \\ Ganesh Chandra Gayen
}

Received: 24 January 2012/ Accepted: 22 February 2012/Published online: 4 March 2012

(C) Association of Otolaryngologists of India 2012

\begin{abstract}
To study the incidence of various foreign bodies in patients of different age and sex, attending E.N.T. Department, a retrospective review was done in Department of E.N.T., Burdwan Medical College and Hospital, Burdwan. About 334 patients with foreign body in ENT region presented to Department of E.N.T., Burdwan Medical College and Hospital from October 2008 to September 2010 were included in the study. Foreign body in ENT region is a common problem frequently encountered in both children and adults. Their accurate diagnosis and management without any complications is often challenging.
\end{abstract}

Keywords Foreign body $\cdot$ Ear $\cdot$ Nose $\cdot$ Throat $\cdot$ Coin

\section{Introduction}

Foreign bodies in the nose, ears and the oropharynx are reason for frequent visits to otorhinolaryngology emergency units. According to the literature, foreign bodies are responsible, on average, for $11 \%$ of otorhinolaryngological emergencies [1]. It may cause increased morbidity and mortality. The major issues for this problem are accurate diagnosis, safe and early removal of foreign body without any complications. The present study is a humble attempt

R. Ray $(\bowtie) \cdot$ M. Dutta $\cdot$ M. Mukherjee $\cdot$ G. C. Gayen

Department of E.N.T, Burdwan Medical College and Hospital,

P. O. Rajbati, Burdwan 713104, West Bengal, India

e-mail: drritamray@gmail.com to describe different types of foreign bodies in ear nose and throat region encountered.

\section{Materials and Methods}

This study was carried out in the Department of E.N.T., Burdwan Medical College and Hospital. It comprises of 334 patients with foreign bodies in upper aerodigestive tract attended both OPD and emergencies of E.N.T. Department of Burdwan Medical College and Hospital, successfully managed during the period of October 2008 to September 2010. All the patients were evaluated carefully with thorough history taking and a complete ENT examination after admission. Radiological investigations like $\mathrm{X}$-ray were done when the foreign body was not visible. This was followed by removal of foreign body.

\section{Observation}

In our study, maximum patients fall within the under five age group $(43.8 \%)$ (Table 1). There was a male predominance. Out of 334 cases, 172 cases (52\%) were males and 162 cases $(48 \%)$ were females. Vegetable foreign bodies are commonly impacted inside nasal cavity whereas metallic foreign bodies (Fig. 1) were commonly impacted inside aerodigestive tract. About $31.7 \%$ foreign bodies were radio opaque and $19.4 \%$ foreign bodies showed normal radiograph. In rest $48.9 \%$ of cases, no radiograph was done because in those cases the foreign body was visible to naked eye.

Most common foreign body we encountered was coin (24.85\%) (Fig. 3), followed by fish bone (15.56\%) and vegetable seeds (14.67\%). Then comes the maggots (11.07\%) (Table 2). 
Table 1 Age incidence

\begin{tabular}{lcl}
\hline Age in years & No. of cases & $\%$ \\
\hline $0-5$ & 146 & 43.8 \\
$6-10$ & 60 & 18 \\
$11-20$ & 22 & 6.5 \\
$21-30$ & 23 & 6.9 \\
$31-40$ & 11 & 3.3 \\
$41-50$ & 21 & 6.3 \\
$51-60$ & 19 & 5.6 \\
$>60$ & 32 & 9.6 \\
\hline
\end{tabular}

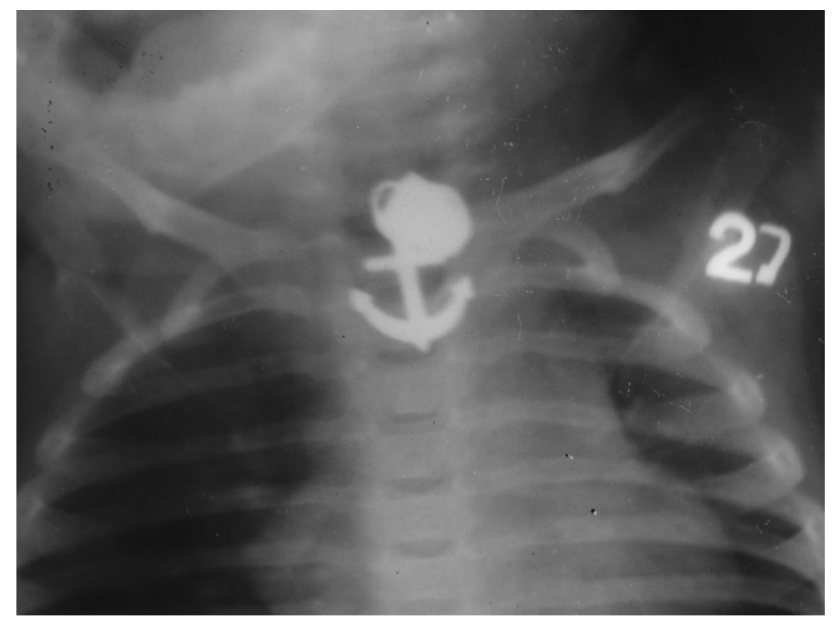

Fig. 1 X-ray showing radio opaque foreign body (metallic locket) in throat

\section{Discussion}

The study of foreign body is fascinating, presenting many regional peculiarities. Otorhinolaryngologists deal with most of the natural orifices that are habitually exposed, such as the mouth, nostrils and ears. The oesophagus and lower airways are affected indirectly, as foreign bodies must first pass through the pharynx or the nasal fossae. Oropharyngeal and nasal fossae foreign bodies are potentially oesophageal and bronchial foreign bodies. Foreign body ingestion is usually accidental, but it may be homicidal or suicidal occasionally.

Foreign bodies may vary widely in shape, size and composition. In our study, the most common age group affected was between 1 and 5 years (Table 1) which correlates with most of the studies [2-5]. Children are mostly affected due to their tendency to take things in their mouth, inability to masticate well and inadequate control of deglutition, as well as tendency to cry, shout and play during eating [6]. In elderly patients, edentulous condition and poor masticating habits are the predisposing factors [6].

Foreign body ingestion is common in children, but frequently seen among intellectually challenged or mentally
Table 2 Types of foreign body

\begin{tabular}{lll}
\hline Type & No. of cases & $\%$ \\
\hline Coin (throat) & 83 & 24.85 \\
Fish bone (throat) & 52 & 15.56 \\
Vegetable. seeds (ear) & 49 & 14.67 \\
Maggot (nose and ear) & 37 & 11.07 \\
Chalk (nose) & 14 & 4.19 \\
Insects (ear) & 13 & 3.89 \\
Meat bolus (throat) & 14 & 4.19 \\
Chicken bone (throat) & 12 & 3.59 \\
Denture (throat) & 11 & 3.29 \\
China battery (nose) & 10 & 2.99 \\
Cycle ball (ear) & 7 & 2.09 \\
Sponge (ear and nose) & 5 & 1.49 \\
Tamarind seeds (ear) & 4 & 1.19 \\
Dot pen cap (throat) & 4 & 1.19 \\
Mutton bone (throat) & 4 & 1.19 \\
Metallic locket (throat) (Fig. 3) & 3 & 0.89 \\
Iron nut (nose) & 3 & 0.89 \\
Metallic ring (throat) & 3 & 0.89 \\
Mango cornel (throat) & 2 & 0.59 \\
Whole beetle nut (throat) & 2 & 0.59 \\
Broken steel spoon (throat) & 1 & 0.29 \\
Safety pin (throat) & 1 & 0.29 \\
\hline
\end{tabular}

ill adults also [7]. Foreign body is usually ingested accidentally, but it may be homicidal or suicidal occasionally.

The most common ear foreign bodies include beads, plastic toys, and paddy seed, popcorn kernels. Insects are more common in patients older than 10 years. In many cases, patients with foreign bodies in the ear are asymptomatic, and in children the foreign body is often an incidental finding. Other patients may present with pain, symptoms of otitis media, hearing loss, or a sense of ear fullness. In one series, $3 \%$ of patients required general anaesthesia to facilitate removal of an ear foreign body; the majority of those patients were younger than 7 years.

The most common site of impaction of foreign body inside nasal cavity is between the septum and inferior turbinate which is anatomically narrowed part. Impacted foreign body presents with unilateral foul smelling nasal discharge.

Foreign body in digestive tract presents with dysphagia, foreign body sensation inside throat, odynophagia and pooling of saliva. In our study, coin is the most common foreign body (Table 2) found inside the throat which is similar to Sam et al. [5] and commonest site of lodgement is the cricopharynx. Coin (Fig. 3) being the daily legal tender, go to the hands of the toddlers and children and they accidentally swallow it. Apart from meat bone (Fig. 2), meat bolus is also not uncommon and typically 


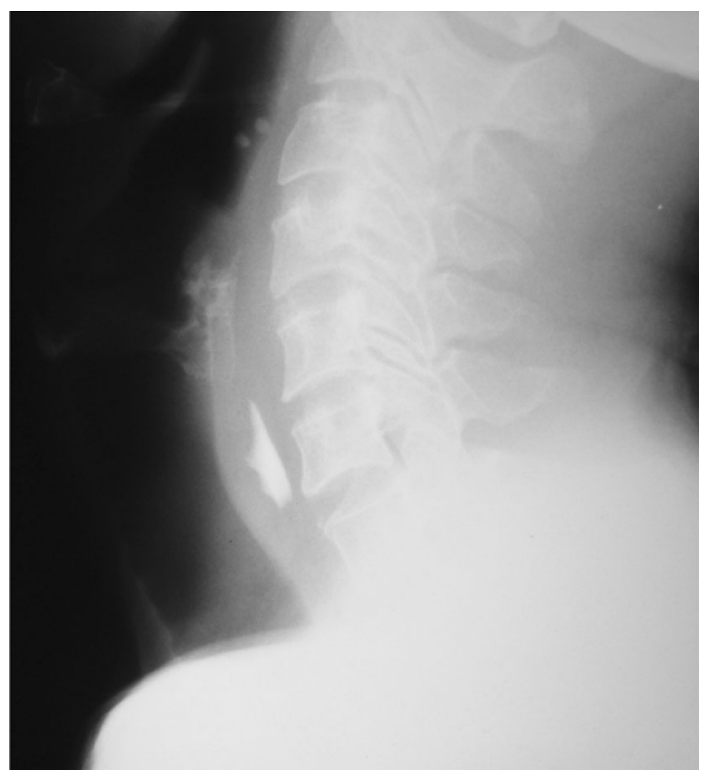

Fig. 2 X-ray showing radio opaque foreign body (meat bone) in throat

seen in patients over 60 years of age and they come in clusters following religious festivals. The cause of impaction of meat bolus in oesophagus may be the defective peristalsis due to age related neuromuscular incoordination.

Radiological investigations like X-ray, CT scan, and MRI are very useful diagnostic tool. In our study we advised X-ray in patients whose foreign bodies are not visible from outside. CT scan and MRI were not required in any case in this study. CT scanning and MRI are rarely useful in the evaluation of foreign bodies in the aerodigestive tract, but are indicated where the object is not found during endoscopic examination and migrated extraluminally and where it is present is unusual and difficult to reach areas. In cases, where the foreign body is metallic specially bullet and present around the skull base, MRI should not be done due to the risk of intracranial migration.

Foreign bodies from the digestive tract are usually removed by esophagoscopy using rigid fiberoptic esophagoscope under general anaesthesia as we have done in our study. But flexible fiberoptic scope can also be used. It easily helps to detect the site of impaction of foreign body. Sometimes, especially in aged patients with cervical spondylosis where neck extension is not possible, it can be used to remove the foreign bodies. Another method is pushing the foreign body into the stomach with a bougie [8]. Vascular catheters such as fogarty or swan ganz have been used in few cases [9]. Intravenous glucagon as a method of treatment for oesophageal meat impaction has been tried [10].

Sharp foreign bodies of neck like needle, wire etc. may migrate extraluminally as their position changes with each

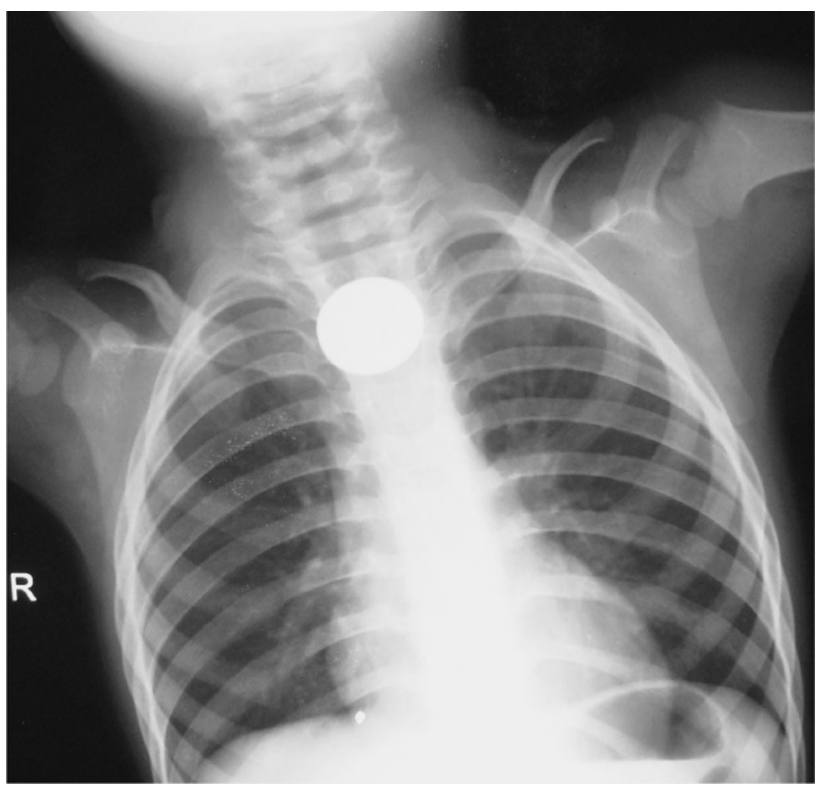

Fig. 3 X-ray showing radio-opaque foreign body (coin) in throat

act of deglutition. During removal of these foreign bodies, per-operative fluoroscopy (C-arm) helps to detect the exact position of foreign body. Fluoroscopically controlled foley's catheter can be used to remove the non-opaque, soft smooth oesophageal foreign bodies like meat bolus [9].

Usual complications related with the foreign bodies are anaesthetic complications. Apart from that, oesophageal perforation, mediastinitis and pulmonary complications are also important complications. Longstanding foreign body may cause erosion of oesophageal wall. Oesophageal stricture is a late complication. Conservative management may be tried failure of which may require surgical interventions thoracotomy etc.

Adequate visualization, appropriate equipment, a cooperative patient and a skilled physician are the keys to successful foreign body removal. The site of impaction, size, and shape of foreign body is important to plan the management protocol.

\section{References}

1. Bressler K, Shelton C (1993) Ear foreign-body removal: a review of 98 consecutive cases. Laryngoscope 103(4.1):367-370

2. Banerjee S (1999) Concept of foreign body, in past and present. Indian J Otolaryngol Head Neck Surg 51(1):23-30. doi: 10.1007/BF03001548

3. Das SK (1984) Aetiological evaluation of foreign bodies in the ear and nose. J Laryngol Otol 98:989-991

4. Higo R, Matsumoto Y, Ichimura K, Kaga K (2003) Foreign bodies in the aerodigestive tract in paediatric patients. Auris Nasus Larynx 30:397-401 
5. Endican S, Ear Joseph P (2006) Nose and throat foreign bodies in Malayasian children: analysis of 1037 cases. Int J Paediatr Otolaryngol 70(9):1539-1545

6. Jyothi AC, Shrikrishna BH, Sanjay G, Sandeep SG, Chaitanya V (2011) A clinical study regarding foreign bodies in aerodigestive tracts. Odisha J Otolaryngol Head Neck Surg 5(1):9-15

7. Sanowski RA (1987) Foreign body extraction in the gastrointestinal tract. In: Sivak MV (ed) Gastroenterological endoscopy. W. B. Saunders Co, Philadelphia, pp 321-331
8. Bonadio WA, Jona JZ, Glicklich M, Cohen R (1988) Esophageal bouginage technique for coin ingestion in children. J Pediatr Surg 23:917-918

9. Nixon GW (1979) Foley catheter method of esophageal foreign body removal: extension of applications. Am J Radiol 132: 441-442

10. Ferrucci JT, Long JA (1977) Radiologic treatment of esophageal food impaction using intravenous glucagons. Radiology $125: 25-28$ 